\title{
Strategies for Knowledge Management in the UK Construction Industry: Benefits and Challenges
}

\author{
Haddy Jallow, Suresh Renukappa, Subashini Suresh and Ahmed Alneyadi \\ Faculty of Science and Engineering, University of Wolverhampton, UK \\ Haddyj 04@outlook.com \\ Suresh.Renukappa@wlv.ac.uk \\ S.Subashini@wlv.ac.uk \\ eng.alneyadi@hotmail.com \\ DOI: 10.34190/KM.19.079
}

\begin{abstract}
In the past there have been no structured approach to gaining information from construction projects once it has been completed which is why Knowledge Management (KM) has received a considerable amount of attention, knowledge in the construction industry is an important resource for an organisation. A clear majority of the construction industry organisations are aware of the benefits that knowledge management can offer, hence construction organisations have invested in knowledge management to achieve those benefits. The purpose of this research is to propose a knowledge management model for construction projects. There are three parts to this paper which are as follows; (1) examine how important Knowledge Management is to the construction industry in the UK; (2) to identify the key resources which can be implemented to benefit KM within an organisation; and (3) to identify the issues and pitfalls that are faced through the implementation stage of KM strategies. The final section of this paper will propose a Knowledge Management model which would benefit construction projects in the UK. The study has found that construction companies in the UK are aware of the benefits of KM, however its implementation is not usually fully completed, the findings of this paper evidently demonstrate that changes to an organisation can lead to a successful KM, the study also indicates a difference between KM in Design and Construction firms.
\end{abstract}

Keywords: knowledge management, construction, implementation, information technology

\section{Introduction}

The construction industry is always looking for continuous improvement and have been implementing various methods which are intended to improve the construction process. These methods include providing different procurement strategies to further improve their communication processes between the different disciplines within the construction organisation (Construction Excellence, 2004). The UK construction industry has resulted in the use of computer technology to utilise an electronic data sharing source in addition to the use of a range of tools and concepts to improve collaboration, quality and efficiency within a project while benefiting construction methods leading to a sustainable construction process (Cherafat, 2010).

Despite all the efforts to improve the construction processes, it is well known that the construction industry struggles to manage project knowledge which can be improved majorly (Design Buildings, 2017). The construction industry is realising that Knowledge Management (KM) can improve the performance of a business which can be beneficial to the industry (Design Buildings, 2017), a construction project contains a lot of knowledge data in the form of paper which can eventually get lost due to a poor filing system and in the construction industry people move onto other jobs where the project can loose information due to a loss of employees at a project, this lead to wasted activities and can affect the project performance.

This paper aims to identify the key resources including information technology which can improve Knowledge management and issues that can be faced while trying to implement the strategies with these resources. This research paper contributes to Knowledge Management in the construction industry and is developed with the aid of academia research studies based on KM. The research begins with a definition of KM and how important it is to the construction industry. Finally, a knowledge management model which can enable organisations in the construction industry to implement knowledge management strategies the right way to assist them in improving their business performance.

\section{Knowledge management}

Knowledge management can be defined as a process of actively managing assets and their data that can create value in an organisation, increase project productivity and capture all the organisations data in an innovative 
manner (Turner, Zimmerman, \& Allen, 2012). KM involves capturing and re-using project knowledge data within an organisation.

\subsection{Defining knowledge}

Understanding the term "knowledge" is a vital part of achieving an affective knowledge management process. There are different forms of knowledge, if knowledge was interchangeable, this then would focus on information exchange and management of information (Samrtsheet, 2019).

Knowledge can be defined as a human's justification to the truth to understand the why, how and who (Bratianu, 2018). It is necessary to know that knowledge can become very valuable based on its final use which assist sustainability over time. Knowledge is built from data which can be then processed into information which is then transformed into knowledge once validated (Bratianu, 2018).

\subsubsection{Knowledge types}

There are two main types of KM which are mostly identified. Explicit knowledge; this is gaining knowledge which can be easily articulated and understood hence it is easily transferred between people. Explicit knowledge can be in the form of a written instruction or a math equation (Samrtsheet, 2019). The second main form of KM is Tactic Knowledge; this form of knowledge is the opposite to explicit knowledge, it is one which the information is not easily shared with others, they are usually skill sets that are challenging to teach others such as innovative thinking (Samrtsheet, 2019). In addition to these two main types of knowledge, there are other forms of knowledge which are as follows:

- Factual Knowledge: This form of knowledge is a measurable one, it can be gained through an observation to verify data.

- Conceptual Knowledge: Conceptual knowledge is based on systems and perspectives.

- Expectational Knowledge: This type of knowledge is mainly derived from expectations or judgements.

- Methodological Knowledge: This deals with solving problems and decision making.

\subsection{Management of knowledge}

Managing knowledge involves a variety of different tasks to ensure that the knowledge is being generated, transferred and being stored for future purposes. This process involves a utilisation of infrastructure tools which can be not straight forward (Liebowitz, 1999). To implement a successful KM process, there are a few factors that are to be considered which are as follows;

- Project knowledge is to be managed which includes project data and knowledge. It is also necessary to identify what the knowledge brings to the project and whom it affects.

- It should also be noted as to how the knowledge is put into use and at what stage does it benefit the project.

- How this knowledge is captured is also a main factor as it indicates how easily knowledge will be shared within an organisation.

- The managed knowledge in the organisation should be monitored to improve the businesses performance.

Processes of KM refer to the processes involved with managing the knowledge within and project or organisation. This paper will focus on the issues that have been found on the tool being used for Knowledge management in the UK infrastructure sector and a discussion on KM at an organisational level in the UK Construction industry.

\section{KM and UK construction organisations}

Performance in the UK construction industry has a major part in the organisations businesses (Davis \& Wilen, 2017). The construction industry is widely based on solving problems in a short amount of time while saving costs, hence why senior construction operatives are becoming more aware that management practices count towards the business (Davis \& Wilen, 2017).

The construction industry uses Key Performance Indicator's (KPI's) to measure performance which fits in well with Knowledge Management. Organisations in the UK construction industry usually provide a framework for 
decision making (Chen, 2015), without a KM strategy, an organisation can have tough boundaries to face. Knowledge Management is quite a new concept to the construction organisations as they are understanding that $\mathrm{KM}$ is a major necessity for asset maintenance.

In the UK Knowledge Management is not practiced very well as most projects are usually Joint ventures between two or more organisations. Sharing information and managing Knowledge within the projects and organisations processes are usually not put into place which lead to loss of information or reduced productivity due to lack of knowledge sharing. Knowledge within a project mainly the individuals within the project team, however this is not captured for future use to better future projects (Takhtravanchi, 2015). Researchers Morteza ShokriGhasabeh (Shokri-Ghasabeh, 2014) have stated this theory indicating the Knowledge is not integrated well with the project teams, in other words, the process of capturing knowledge, sharing and reusing within a project and organisation is leading to low project performance.

\section{Knowledge management strategies in the UK construction industry}

In the UK, there are more than 1.5 million employees, the industry takes a massive part of the overall GDP (Gross Domestic Product) (Construction 2025, 2013). Eagan (Egan, 1998), the UK construction industry has been affected as there have been performance issues which have been ongoing. Within the report Egan pointed out that there are two main factors that can benefit the performance of the UK construction industry, knowledge and learning.

$\mathrm{KM}$ is becoming more popular in the construction industry and as mentioned before it is a critical part of organisations to gain some competitive edge. Projects can be unique and complicated and with temporary team member in the project all knowledge captured while they are present would be a huge asset to the project itself. Despite the awareness of KM, there is still little understanding on ways in which knowledge can be captures and shared in the best way. Soliman (Soliman \& Spooner, 2000) have argued that when it comes to KM, the people in the organisation are to clearly state all relating aspects to KM.

Within the construction industry in the UK, there is a lot of skill and labour required which means it is heavily people based hence the best way of sharing knowledge is to adopt a KM strategy and spread it amongst the people to enhance performance throughout the organisation. There are numerous KM approaches that are currently being adopted within organisations and all their approaches have strengths and weaknesses. There are two main activities trending on KM:

- Technology tools are becoming more and more popular in the construction industry, with the Industry revolution 4.0, several KM solutions are being identified and the Information Technology assist in this as when it comes to knowledge, asset technologies are being created to manage knowledge within organisations.

- People track knowledge management is the second trend. This focuses on the behavioural approach of the organisation.

The two trending tracks of KM take two different outlooks, the first trend is based on the management of information (Sveiby, 2002). This trend focuses on the use of information management systems, artificial intelligence and cloud-based forms of managing information. This trend is the new generation of KM however this trend requires people involved within a project to be proactive with the Information technology systems used within their organisation and with people being used to previous processes this tends to be difficult.

There are a few different theories as to KM approaches, Pan and Scarbrough (Pan \& Scarbrough, 1999) state that there are two approaches to KM which are noted to be as follows:

- Supply driven: The supply driven approach focuses on the issues surrounding Knowledge Management and the sharing of knowledge within an organisation. This approach aims to increase KM by capturing information and transmitting knowledge with the use of technology.

- Demand driven: This approach is more focused on the users concern and motivation and what attitude they have towards capturing knowledge.

Other approaches to KM are discussed by Hansen et al (Hansen, 1999) where KM is split into two different categories, Codification and Personalisation. 
- Codification: This discusses the use of databases where knowledge can be shared within the organisation, the focus of this approach is having available knowledge stored and accessible for use at a prompt access to the knowledge being shared.

- Personalisation: This strategy highlights the process of sharing knowledge through personal contacts, however this form of knowledge sharing is known to be informal whereas the codification approach is a formal method which involves the use of electronic databases.

\section{Different benefits of the formal and informal approaches to KM in construction}

Knowledge Management in an organisation is known to improve a projects performance improving quality, time and saving costs (Armistead, 1999). There have been several researches published highlighting the benefits that can be gained through KM. KM is known to enhance collaboration within an organisation and the sharing of knowledge allows people to express themselves within an electronic environment. KM assists in saving business problems and helps increase benefits through the following ways:

- Promoting best practices through improving the business decision making.

- Enhancing productivity and efficiency by adopting a smarter way of working.

- Driving innovative ideas across the organisation while improving collaboration.

- Capturing knowledge for future use, saving loss of time through the know how of the stored knowledge.

- Improving client satisfaction by providing insight to the project at hand.

- Improving quality and the ability to collaborate and enhance discussions between the experts (Deloitte, 2019).

All these benefits can save costs or enable the organisation to earn money, however many of these benefits are difficult to quantify (McCampbell, 1999).

\section{Challenges found with knowledge management within the UK construction industry}

The construction industry has yet to fully gain these available benefits from KM to improve the organisations performance. There are four main barriers that have been outlined in previous research as to why the construction industry is yet to gain these benefits which are as follows; organisations current culture, lack of implemented and adopted work practices, costs and not enough time (Carillio, 2006). Through the review of literature the following major barriers were found within the industry:

\subsection{Social barriers}

- People related: Tactic knowledge is usually apprehended through networking with the project team in an informal way. This knowledge is then not captured and relayed to the rest of the team and individuals will collect the knowledge from professionals and with the overwhelming information it is not usually shared.

- Culture: one of the main barriers faced in the UK is culture, experts in certain fields are usually reluctant to share knowledge that has been captured as the more they know, the more powerful they can be compared to others.

- Motivational barriers: Project managers can generally increase awareness within the team in order to achieve better KM and voice the importance of capturing and sharing knowledge, however this is not the current case in the UK construction industry.

\subsection{Technical barriers}

Knowledge capturing is currently mainly focused on the use of IT software, however even with the provision of these software's, the UK construction industry lacks technical support as most employees are from an older generation whom are used to the non-IT ways of capturing knowledge (BenMoussa, 2009).

Other challenges face are described below:

Lack of time: Capturing knowledge and sharing it can be time consuming, and with construction projects always working to tight deadlines, anything distracting the main business can seem to be of low importance. 
Converting knowledge: A main challenge which spreads across the construction industry is how organisation capture knowledge. There are skilled individuals and workers within the industry with masses of experience, however converting this knowledge from the skilled workers to benefit others is a major issue.

Unique projects: the UK Construction industry usually utilise knowledge gained while working on a project, this knowledge however is not transferred to follow on projects and essentially KM is wasted as the following project may be different.

Time consuming: KM is a long-term goal which requires time in the beginning stages of the strategy. Organisations may see this time and effort put in at the early stages to be a waste and lose faith in the process.

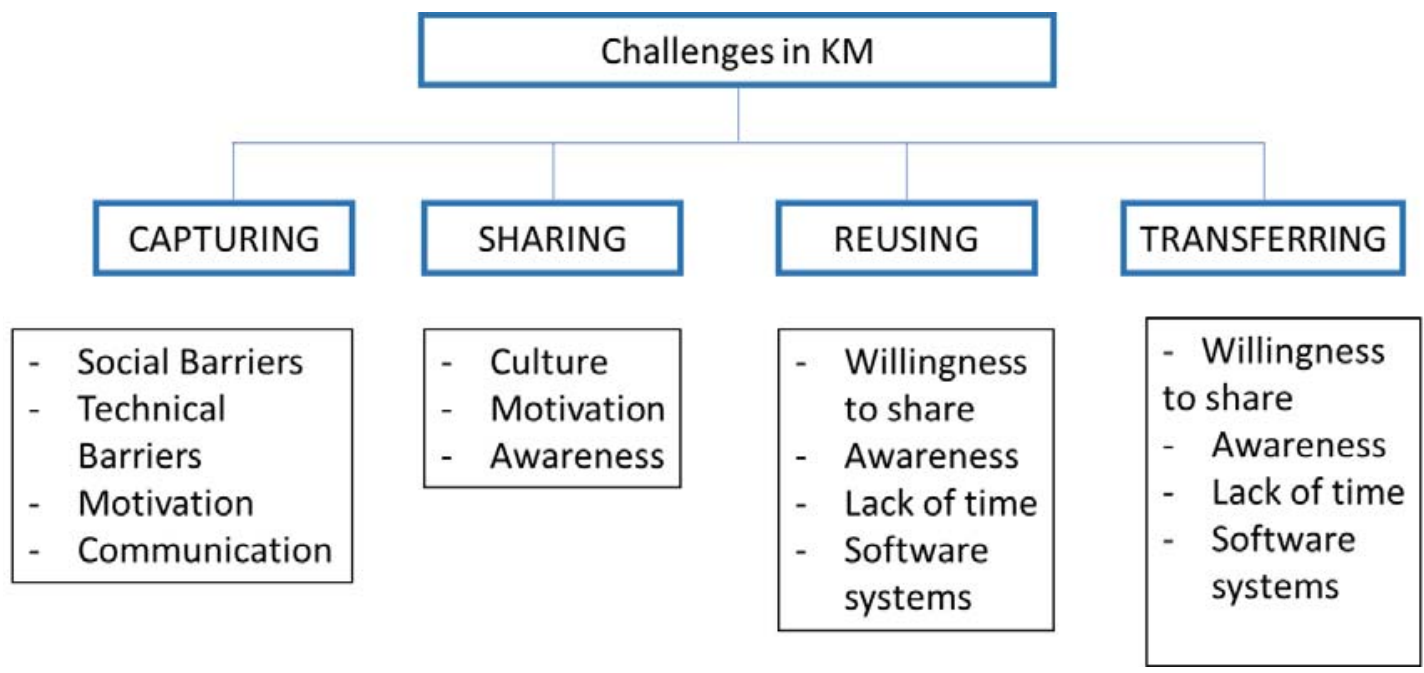

Figure 1: Knowledge process challenges

\section{Recommendations for the industry}

A knowledge management strategy can provide opportunity for the organisation to understand the challenges that are being faces and how to solve them. Based on the literature, there are a few issues on adopting a KM strategy, suggestions below can assist in the potential success of a KM strategy.

\subsection{Change of management plan}

$\mathrm{KM}$ in the construction industry means a cultural change to the way that people in the industry are used to working. Employees are to get on board onto the KM strategy which may change their way of working. A change of the management plan would specify to workers how to accept the KM processes within the organisation (Grayson \& O'dell, 1998).

\subsection{Reward system for encouragement}

The sharing of knowledge is not a natural process, hence when implementing a knowledge management process, a reward system put in place should be established. Recognition in the workplace shows appreciation of the efforts put in by the employees, encouraging them to further share knowledge. This however can become a gaming system to employees where the reward would be the end goal. The reward system not being a longterm process can assist with the gaming mind set, rewarding employees can create interest and excitement in KM (Grayson \& O'dell, 1998).

\subsection{Introduce technology platform}

KM can benefit from having Information Technology involved. IT tool are a very useful asset as the use of technology can enable easier knowledge sharing and capturing. Tactic and explicit knowledge can be created and retained within a technology database leading to knowledge being easily shared through an efficient manner (Grayson \& O'dell, 1998). 


\section{Haddy Jallow et al.}

\subsection{Training}

The construction industry contains employees that have been in the business for many years who are used to the way they have been practicing their day to day job in the industry. For a knowledge management strategy to be implemented and adopted, a training strategy can assist in the implementation of the KM strategy. Through workshops and training sessions employees can learn how to adopt the best practices and it will also promote the human interactions which benefit knowledge sharing.

\section{Conclusion}

This paper has discussed the issues surrounding Knowledge management in the UK construction industry. An introduction to KM was firstly established and how it implicates organisations. It is clear from the discussions that the construction industry needs better KM within organisations to enhance performance of projects and organisations. There are many barriers which need to be overcome to be able to implement a KM strategy within an organisation, the construction industry in the UK is filled with the older generation whom are used to their ways of working and with a lot of staff being temporary, knowledge can be unshared which could benefit following projects. Ways in which to overcome these issues are not lacking, there are a lot of technologies which can assist in capturing knowledge and sharing between employees. Based on this research, KM strategies have become a main asset to the construction industry. To further this research, a more in-depth study into the construction industry in the UK is to be conducted including ongoing study within Knowledge Management which would involve developing a Knowledge Management framework strategy and conducting a main study.

\section{References}

Armistead, C. (1999). Knowledge management and process performance. Journal of Knowledge Management, 143-157.

Bratianu, E. B. (2018). The Elusive Definition of Knowledge. Emergent knowledge strategies: Strategic thinking in knowledge management, 1-22.

Carillio, P. (2006). Exploiting knowledge management: the engineering and construction perspective. Journal of Management in Engineering, 2-10.

Chen, K. (2015, September). A Strategic Decision Making framework for Organisational BIM implementation. Retrieved from Cardiff University: https://orca.cf.ac.uk/88267/1/2016ChenYPhD.pdf

Cherafat, R. R. (2010). Collaboration: a better way to quality, efficiency and value in construction. Laboratory Equipment, 13.

Construction 2025. (2013). Industrial Strategy: government and industry in partnership. London: HM Government.

Construction Excellence. (2004). Procurement. London: Construction Excellence.

Davis, R., \& Wilen, A. (2017). UK Industry Performance Report. watford: Constructingexc.

Deloitte. (2019, March 23). Benefits of knowledge management. Retrieved from Deloitte: https://www2.deloitte.com/lu/en/pages/strategy/articles/benefits-knowledge-management.html

Design Buildings. (2017, July 13). Knowledge Management in Construction. Retrieved from Design Building Wiki: https://www.designingbuildings.co.uk/wiki/Knowledge management in construction

Egan, J. (1998). Rethinking Construction. London: Crown Copyright.

Grayson, C. J., \& O'dell, C. (1998). If Only We Knew What We Know: Identification and Transfer of Internal Best Practices. California Management Review, 154-174.

Hansen, M. T. (1999). The Search-Transfer Problem: The Role of Weak Ties in Sharing Knowledge across Organization Subunits. Administrative Science Quarterly, 82-111.

Liebowitz, J. (1999). Key ingredients to the success of an organization's knowledge management strategy. Knowledge and process management; the journal of corporate transformation, 37-40.

McCampbell, A. S. (1999). Knowledge management: the new challenge for the 21st century. Journal of Knowledge Management, 172-179.

Pan, S. L., \& Scarbrough, H. (1999). Knowledge Management in Practice: An Exploratory Case Study. Technology Analysis \& Strategic Management, 359-374.

Samrtsheet. (2019). What is Knowledge Management. Retrieved from Smartsheet: https://www.smartsheet.com/knowledge-management-101

Shokri-Ghasabeh, M. (2014). Knowledge management: Barriers to capturing lessons learned from Australian contractors perspective. Construction Innovation, 108-134.

Soliman, F., \& Spooner, K. (2000). Strategies for implementing knowledge management: role of human resources management. Journal of Knowledge Management, 337-345.

Sveiby, K.-E. (2002). Collaborative climate and effectiveness of knowledge work - an empirical study. Journal of Knowledge Management, 420-433.

Takhtravanchi, M. (2015). The importance of tacit knowledge integration within traditional Project Environment: A Critical Review. IPGRC, 1-14.

Turner, J. R., Zimmerman, T., \& Allen, J. (2012). Teams as a Process for Knowledge Management. Journal of Knowledge Management, 1-32. 